\title{
VITAMIN D TRENDS IN THE PEDIATRIC ORTHOPAEDIC POPULATION: A SURVEY
}

\author{
Kevin A. Williams, MD' ${ }^{1}$, Michael Horan, MD MS $^{2}$ \\ ${ }^{1}$ Palmetto Health-USC School of Medicine, Columbia, SC, USA, ${ }^{2}$ Palmetto Health, USA \\ Background: \\ Vitamin D deficiency is a global issue, affecting adults and children of all ages, races and genders. \\ Within the last decade, multiple studies have demonstrated the potential health benefits of vitamin \\ D supplementation including improved bone health, reduced fracture risk, protection from \\ autoimmune disease, and decreased cancer risk. Because of the prevalence of vitamin $D$ \\ deficiency in pediatric populations despite recent evidence of increased vitamin $D$ supplementation \\ in the US, our goal is to assess the knowledge of current vitamin D recommendations among \\ Pediatric Society of North America (POSNA) members. It is our purpose to use the data to \\ increase awareness and understanding of vitamin $D$ amongst all pediatric providers.
}

\section{Methods:}

Our survey was distributed to 1316 POSNA members via a series of 2 email requests in which they were invited to participate in the survey on the Survey Monkey website. Their participation was entirely voluntary and they agreed to participate by responding positively on the first page of the survey. The data was depersonalized and analyzed via chi square and Fisher's exact testing.

Results:

395 responses were recorded. 9 participants opted out of the survey. $69 \%$ of participants rated their vitamin $\mathrm{D}$ knowledge as fair to good. $68 \%$ of participants have been in practice over ten years and represented most US geographic regions fairly equally. Most estimate that over $25 \%$ of their practice is vitamin D deficient with about a $50 \%$ compliance rate of supplementation. Over $30 \%$ of participants feel vitamin D management is mostly the role of the pediatrician, however $64 \%$ of participants discuss or check vitamin D levels in their practice for patients with repeat fractures, medical comorbidities, or nonunions most commonly.

\section{Conclusion:}

Survey participants demonstrated a wide variety of responses indicating their understanding of vitamin $\mathrm{D}$ testing and supplementation. Although providers estimate a high deficiency rate, many do not routinely check vitamin D. When they do check, there is no standard indication for testing or supplementation and many believe this to be the role of the pediatrician or endocrinologist. More studies are needed to provide a standardized protocol for vitamin D testing/supplementation in the pediatric orthopaedic literature.

\footnotetext{
This open-access article is published and distributed under the Creative Commons Attribution - NonCommercial - No Derivatives License (http://creativecommons.org/licenses/by-nc-nd/4.0/), which permits the noncommercial use, distribution, and reproduction of the article in any medium, provided the original author and source are credited. You may not alter, transform, or build upon this article without the permission of the Author(s). For article reuse guidelines, please visit SAGE's website at http://www.sagepub.com/journals-permissions.
} 


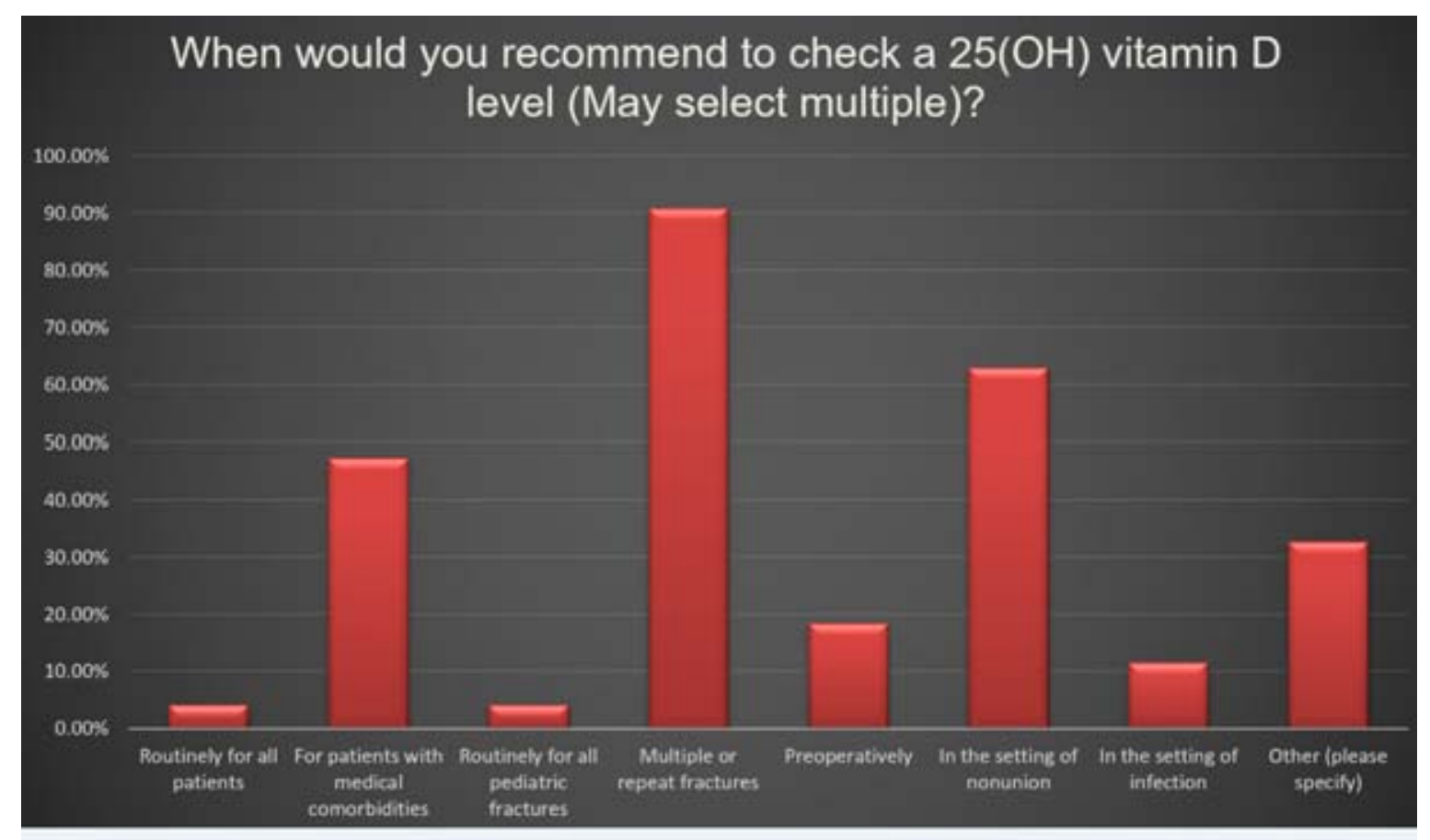

The Orthopaedic Journal of Sports Medicine, 7(3)(suppl 1) DOI: $10.1177 / 2325967119500134$

(CThe Author(s) 2019 